\title{
Low quality evidence on the stability of orthodontic mini-implants
}

\author{
Abstracted from \\ Papageorgiou SN, Zogakis IP, Papadopoulos MA. \\ Failure rates and associated risk factors of orthodontic miniscrew implants: a meta-analysis. \\ Am J Orthod Dentofacial Orthop 2012; 142: 577-595. e7. doi: 10.1016/j.ajodo.2012.05.016. \\ Address for correspondence: Moschos A. Papadopoulos, Department of Orthodontics, \\ School of Dentistry, Aristotle University of Thessaloniki, GR-54124 Thessaloniki, Greece. E-mail: mikepap@dent.auth
}

\section{Question: What are the failure rates of mini- screws used for orthodontic anchorage?}

Data sources Medline, Embase, LILACS, Cochrane Library, Google Scholar, Web of Science, African Journals Online, Digital Dissertations. Study selection Randomised controlled trials (RCTs), prospective controlled clinical trials(CCTs) and prospective cohort studies were included. Studies on implants with a diameter greater than $2 \mathrm{~mm}$ were excluded. Data extraction and synthesis Data were extracted independently by two authors. Bias in RCTs was assessed using the Cochrane risk of bias tool, with non-randomised studies being assessed using the NewcastleOttawa Scale. Failures of mini-screw implants were expressed as event rates with 95\% confidence intervals. Risk factors were assessed using random effects model and sub-group and meta-regression analyses were also conducted..

Results Fifty-two studies; five RCTs, eight CCTs, twenty-seven prospective cohort studies and twelve studies with unclear designs that were assessed to be prospective cohort studies were included. A funnel plot suggested there may be publication bias. Analysis of 4987 miniscrew implants used in 2281 patients indicated an overall failure rate of $13.5 \%$ (95\% confidence interval, $11.5 \%-15.8 \%$ ). Failures were not associated with patient sex or age and mini-screw implant insertion side, whereas they were significantly associated with jaw of insertion. Some trends were identified through exploratory analysis but no definite conclusions could be drawn.

Conclusions Orthodontic miniscrew implants have a modest small mean failure rate, indicating their usefulness in clinical practice. Although many factors seem to affect their failure rates, the majority of them still need additional evidence to support any possible associations.

\section{Commentary}

In most orthodontic treatment plans some form of anchorage is necessary to control the reciprocal forces of tooth movement. ${ }^{1}$ It is usually obtained by applying a force to a group of teeth or through extra-oral sources, eg the neck or cranium. However, these techniques have a limited area of application, may still cause unintended loss of anchorage, or depend on the collaboration of the patient. ${ }^{1}$ Orthodontic mini implants are not conditioned by most of these limitations, are indicated for a wide variety of treatment mechanics and can be used in both jaws over a wide range of time periods. ${ }^{2}$

The failure rate of these devices during the application of orthodontic forces is an important-patient outcome. This issue is addressed in the systematic review that is critically appraised in this commentary. The completion of this broad-spectrum review is a major undertaking and the authors have to be complimented for their persistence. However, I want to discuss some limitations of this article that should have been addressed during the preparation of the protocol and the reviewing and editorial processes. The AMSTAR $^{3}$ and CASP (www.casp-uk.net accessed April 25 $5^{\text {th }} 2013$ ) tools were consulted for this critical appraisal.

Most of the limitations of this systematic review are the result of an inadequate framing of the review question. This paper addressed one broad-spectrum question on the failure rates of miniscrews and over 40 'narrow-spectrum questions' on individual variables that could influence these outcomes. This magnitude of questions alone creates manageability issues for both the review team and the editors. ${ }^{4}$ Broad-spectrum questions are indicated when it is plausible that outcomes are more or less the same for different subpopulations of patients and interventions, but earlier systematic reviews have shown that this is not the case. ${ }^{5,6,7}$ This paper could still have been framed around a broad-spectrum review question that shows the heterogeneity in failure rates between subpopulations, but should then be complemented with only a few a priori well defined narrow-spectrum questions.

The materials and methods section of a systematic review should include a specific chapter 'Criteria for including studies for this review', in which the types of participants, interventions, outcomes and studies are defined. ${ }^{4}$ These definitions were either incomplete or missing in this paper and should have also been defined for each narrow-spectrum question. For example the primary outcome 'failure rates of miniscrews' was not defined. This creates confusion, because authors of the selected studies have defined this outcome differently or not at all. ${ }^{6,8}$ Further, patient-important outcomes such 
as adverse effects of interventions, which are obligatory outcomes in systematic reviews according to the Cochrane collaboration, were not assessed. ${ }^{4}$ The time point for measuring outcomes was also not defined. This is an important factor, because varying durations could introduce heterogeneity, and studies too short in duration could have little relevance. In addition, study selection was based on study design labels and not on explicit design features. To avoid ambiguity, the Cochrane collaboration recommends against using design labels for selecting studies. ${ }^{9}$

Because the narrow-spectrum research questions and their eligibility criteria were not specifically defined, it is impossible to understand why certain studies were selected and how to assess the validity of their outcomes. It is for example not clear why only two studies were selected for assessing associations between insertion torque and failure rates when at least seven of the included articles addressed such an association. ${ }^{10} \mathrm{~A}$ table with the names of the excluded studies and the reasons for exclusion would have been helpful. This lack of transparency is problematic for readers, guideline developers and for researchers who want to update or replicate all or some parts of this paper.

In addition, outcomes of both randomised-controlled trials (RCTs) and non-randomised studies were pooled in the same forest plot. However, when evidence is identified in different study designs, it is favoured to synthesise their effect estimates separately. ${ }^{9,11}$ The presentation of the diamond in the forest plot that summarises the outcomes of all study designs should have been avoided, because it will further mislead the reader. Separate forest plots for each type of study design should have been shown.

To be useful for clinicians, systematic reviews must not only provide an outcome, but also give the necessary information to judge whether this effect estimate is likely to be correct. ${ }^{12}$ These judgments are not based exclusively on the assessment of risk of bias. The GRADE Working Group has identified five categories; risk of bias, imprecision, inconsistency, indirectness and publication bias, which are used to make quality ratings of outcomes. ${ }^{12}$ This rating procedure has been adopted by the Cochrane Collaboration, ${ }^{13}$ but was not conducted in this systematic review. These assessments should have been done separately for RCTs and observational studies. ${ }^{14}$ GRADE provides a starting quality rating, 'high quality', for outcomes from the former and 'low quality' for those from the latter type of studies. ${ }^{12}$ These ratings can be subsequently up- or down-graded based on the application of the five quality criteria.

The quality of the outcome 'implant failure rates' for the broadspectrum review question in the five RCTs could be down-rated to 'low' quality, because of bias, imprecision and 'substantial inconsistency'. Because no information on adverse effects was provided, these quality judgments cannot be placed into perspective. The same three domains could also down-rate the quality of the observational studies to 'very low' quality. Publication bias could probably further negatively affect these ratings. Some of the selected studies were also identified as 'suspect of multiple publication bias' in another systematic review. ${ }^{10}$

The quality ratings of the outcomes of the narrow-spectrum questions are not possible because specific PICOT (Participants, Interventions, Comparisons, Outcomes and Time periods) questions were not defined, and most information to make such judgments was not provided by the authors.
In the conclusions the authors state that 'the small mean failure rates of miniscrews indicate their usefulness in clinical practice'. However, systematic reviewers have been discouraged to make such practice recommendations, because these judgments do not depend on just one patient-important outcome, but also on, eg, the quality of the evidence, the balance between desirable and undesirable outcomes, costs, the setting and input from pertinent stakeholders. ${ }^{12}$

Many of the limitations outlined in the previous sections could have been prevented in the protocol phase of this systematic review. Splitting the article up in several different publications with narrower-spectrum PICOT questions could have made this broad-spectrum review more manageable and accessible. ${ }^{4}$ A methodologist with expertise in systematic reviewing and conducting meta-analyses should have been included in the review team to assist with methodological issues.

However, also the editors and peer-reviewers of the American Journal of Orthodontics and Dentofacial Orthopedics (AJODO) have an important impact on the quality of this paper. This article for example is not structured according to a validated format for reporting systematic reviews. ${ }^{15,16,17}$ Although the AJODO has adopted the PRISMA statement and promotes the protocols of the Cochrane Collaboration for reporting systematic reviews, full endorsement of these reporting formats in this journal has been deficient. ${ }^{18,19,20}$ The quality of systematic reviews will only improve when editors implement these guidelines and assess whether authors have complied with these protocols as well. ${ }^{21}$ Peer-reviewers and editors owe such rigour to the hard work of current and future review teams. Some minor suggestions by these stakeholders could have significantly improved the quality of this paper.

Failure rates of orthodontic mini implants in combination with other patient-important outcomes should be evaluated when implementing these devices for orthodontic anchorage. True failure rates in different subpopulations of patients and interventions will probably vary substantially from those summarised in the forest plot of this systematic review.

\section{Practice points}

- The critical appraisal of this systematic review suggests that the quality of the body of evidence on failure rates of orthodontic mini-implants is low

- Editors of journals should not only adopt validated reporting guidelines of systematic reviews, but should also implement them.

\section{Reint Meursinge Reynders Private Practice, Milan, Italy}

1. Skeggs RM, Benson PE, Dyer F. Reinforcement of anchorage during orthodontic brace treatment with implants or other surgical methods. Cochrane Database Syst Rev 2007 Jul 18;(3):CD005098.

2. Papadopoulos MA, Papageorgiou SN, Zogakis IP. Clinical effectiveness of orthodontic miniscrew implants: a meta-analysis. J Dent Res 2011; 90: 969-976.

3. Shea B], Grimshaw JM, Wells GA, et al. Development of AMSTAR: a measurement tool to assess the methodological quality of systematic reviews. BMC Med Res Methodol 2007; 7: 10.

4. O'Connor D, Green S, Higgins JPT (editors). Chapter 5: Defining the review question and developing criteria for including studies. In: Higgins JPT, Green S (editors), Cochrane Handbook of Systematic Reviews of Intervention. Version 5.1.0 (updated March 2011). The Cochrane Collaboration, 2011. Available from www.cochranehandbook.org (Accessed August $13^{\text {th }}$ 2013).

5. Guyatt GH, Oxman AD, Kunz R, et al. GRADE guidelines: 2. Framing the question and deciding on important outcomes. J Clin Epidemiol 2011; 64: 395-400. 
6. Reynders R, Ronchi L, Bipat S. Mini-implants in orthodontics: a systematic review of the literature. Am J Orthod Dentofacial Orthop 2009; 135: 564. e1-19.

7. Schätzle M, Männchen R, Zwahlen M, Lang NP. Survival and failure rates of orthodontic temporary anchorage devices: a systematic review. Clin Oral Implants Res 2009; 20: 1351-1359.

8. Papageorgiou SN, Zogakis IP, Papadopoulos MA. Failure rates and associated risk factors of orthodontic miniscrew implants: a meta-analysis. Am J Orthod Dentofacial Orthop 2012; 142: 577-595. e7.

9. Reeves BC, Deeks JJ, Higgins JPT, Wells GA. Chapter 13: Including non-randomized studies. In: Higgins JPT, Green S (editors), Cochrane Handbook for Systematic Reviews of Interventions Version 5.1.0 (updated March 2011). The Cochrane Collaboration, 2011. Available from www.cochrane-handbook.org (Accessed August $13^{\text {th }}$ 2013).

10. Meursinge Reynders RA, Ronchi L, Ladu L, van Etten-Jamaludin F, Bipat S. Insertion torque and success of orthodontic mini-implants: a systematic review. Am J Orthod Dentofacial Orthop 2012; 142: 596-614. e5.

11. Valentine JC, Thompson SG. Issues relating to confounding and meta-analysis when including non-randomized studies in systematic reviews on the effects of interventions. Res Syn Meth 2013; 4: 26-35.

12. Balshem $H$, Helfand $M$, Schünemann $H$ J, et al. GRADE guidelines: 3 . Rating the quality of evidence. / Clin Epidemiol 2011; 64: 40-406.

13. Schünemann $H$ J, Oxman $A D$, Vist GE, Higgins JPT, Deeks II, Glasziou P, Guyatt GH Chapter 12: Interpreting results and drawing conclusions. In: Higgins JPT, Green S (editors), Cochrane Handbook for Systematic Reviews of Interventions Version 5.1.0 (updated March 2011). The Cochrane Collaboration, 2011. Available from www. cochrane-handbook.org (Accessed August 13 ${ }^{\text {th }}$ 2013).

14. Guyatt GH, Oxman AD, Santesso N, et al. GRADE guidelines: 12. Preparing summary of findings tables-binary outcomes. J Clin Epidemiol 2013; 66: 158-172.

15. Liberati A, Altman DG, Tetzlaff J, et al. The PRISMA statement for reporting systematic reviews and meta-analyses of studies that evaluate health care interventions: explanation and elaboration. PLoS Med. 2009; 6: e1000100. Epub 2009 Jul 21.

16. Higgins JPT, Green S (editors). Cochrane Handbook for Systematic Reviews of Interventions Version 5.1.0 [updated March 2011]. The Cochrane Collaboration, 2011. Available from www.cochrane-handbook.org (Accessed March $13^{\text {th }} 2013$ ).

17. Moher D, Liberati A, Tetzlaff I, Altman DG; PRISMA Group. Preferred reporting items for systematic reviews and meta-analyses: the PRISMA statement. PLoS Med 2009; 6: e1000097.

18. Fleming PS, Seehra J, Polychronopoulou A, Fedorowicz Z, Pandis N. A PRISMA assessment of the reporting quality of systematic reviews in orthodontics. Angle Orthod 2013; 83: 158-163.

19. Kokich VG. Happy 20th anniversary! Am J Orthod Dentofacial Orthop 2013; 144: 1

20. Turpin DL. Updated CONSORT and PRISMA documents now available. Am J Orthod Dentofacial Orthop 2010; 137: 721-722.

21. Fanelli D. Redefine misconduct as distorted reporting. Nature 2013; 494: 149.

Evidence-Based Dentistry (2013) 14, 78-80. doi:10.1038/sj.ebd.6400950 